\title{
The wandering mind oscillates: EEG alpha power is enhanced during moments of mind-wandering
}

\author{
Rebecca J. Compton ${ }^{1} \cdot$ Dylan Gearinger ${ }^{1} \cdot$ Hannah Wild ${ }^{1}$
}

Published online: 9 September 2019

(C) The Psychonomic Society, Inc. 2019

\begin{abstract}
What is your brain doing while your mind is wandering? This study used a within-subjects experience-sampling design to test whether episodes of mind-wandering during a demanding cognitive task are associated with increases in EEG alpha power. Alpha refers to cyclic oscillations in EEG activity at $8-12 \mathrm{~Hz}$, and has been previously correlated with internally rather than externally directed cognition. Participants completed a speeded performance task with more than 800 trials while EEG was recorded. Intermittent experience-sampling probes asked participants to indicate whether their mind was wandering or on-task. Participants reported mind-wandering in response to approximately half of the probes. EEG alpha power was significantly higher preceding probes to which participants reported mind-wandering, compared with probes to which participants reported being on task. These findings imply that dynamic changes in alpha power may prove a valuable tool in studying momentary fluctuations in mind-wandering.
\end{abstract}

Keywords Mind-wandering $\cdot$ EEG $\cdot$ Alpha oscillations $\cdot$ Experience sampling

Mind-wandering is a common occurrence, but it is challenging to study because it is internally experienced and spontaneously generated. Sometimes referred to as daydreaming, task-unrelated thought, or off-task cognition, mindwandering generally refers to cognition that is directed toward an internal stream of thought rather than toward external stimuli or tasks immediately at hand (Smallwood \& Schooler, 2015). A continuing methodological challenge is identifying when mind-wandering episodes occur, as cognition unfolds on a dynamic moment-to-moment basis.

Measures of brain activity could provide indices of mindwandering, as well as information about its neural basis, if such measures could be reliably associated with mindwandering episodes (Gruberger, Ben-Simon, Levkovitz, Zangen, \& Hendler, 2011). Some fMRI studies have found that activity increases in a set of brain regions known as the default mode network during states associated with mindwandering, such as conditions when the participant has no explicit task (resting state versus a task-directed condition) or when the participant reports engaging in more versus less

Rebecca J. Compton

rcompton@haverford.edu

1 Department of Psychology, Haverford College, 370 Lancaster Ave, Haverford, PA 19041, USA spontaneous thought (Andrews-Hanna, Irving, Fox, Spreng, $\&$ Christoff, 2018). Although such fMRI approaches are promising, the prohibitive cost and practical constraints of fMRI, combined with a sluggish hemodynamic response that may not easily track momentary changes leave the door open for complementary approaches.

The temporal precision of EEG makes it a promising technique for studying mind-wandering episodes. Specifically, EEG alpha activity may provide a potential indirect index of internally directed thought. Since the invention of EEG technology in the 1920s, alpha waves, which are patterns of oscillatory EEG activity cycling at $8-12 \mathrm{~Hz}$, have been associated with mental states conducive to mind-wandering. Alpha waves are higher in waking eyes-closed compared with eyes-open conditions (Adrian \& Mathews, 1934). During cognitive tasks, alpha waves are suppressed in response to external stimulation, such as task-relevant visual images or warning cues (e.g., Thut, Nietzel, Brandt, \& Pascual-Leone, 2006). Conversely, alpha waves are enhanced during brief waiting intervals between task trials (Carp \& Compton, 2009; Compton, Arnstein, Freedman, Dainer-Best, \& Liss, 2011) and during mental imagery, which requires inward-directed thought (Cooper, Croft, Dominey, Burgess, \& Gruzelier, 2003). Increased alpha activity prior to an incoming stimulus is associated with decrements in perceptual discrimination (e.g., van Dijk, Schoffelen, Oostenveld, \& Jensen, 2008). 
Finally, alpha-wave activity has been correlated with defaultmode-network activity in joint EEG-fMRI research (Knyazev, Slobodskoj-Plusnin, Bocharov, \& Pylkova, 2011; Mo, Liu, Huang, \& Ding, 2013). Together, these results provide a circumstantial argument that alpha oscillations and mind-wandering may co-occur.

Several prior studies have more directly examined the relationship between mind-wandering and EEG alpha power, with somewhat conflicting results. In one of the first studies to examine this relationship, Braboszcz and Delorme (2011) asked participants to count their own breaths and to press a button when their minds wandered away from this primary task. Contrary to the expectation that alpha power would be positively correlated with mind-wandering, these researchers found the opposite: alpha power was decreased in a window of time prior to a button-press indicating mind-wandering. However, two factors limit interpretation of this study. First, the primary task, breath-counting, involves internally directed attention, which would be expected to increase alpha activity under the assumptions that alpha taps internally versus externally driven attentional focus. Second, the study used a "selfcaught" method of identifying episodes of mind-wandering, in which the event that marked mind-wandering was the participant's meta-awareness that mind-wandering had occurred. Conscious awareness of one's own mind-wandering may tap different mechanisms than mind-wandering itself. For both of these reasons, the findings of Braboszcz and Delorme (2011) may not characterize the neural processes involved when a participant's attention drifts away from an external task and toward an internal train of thought.

Other studies have found, in contrast to Braboszcz and Delorme (2011), evidence that associates increased EEG alpha power with mind-wandering episodes. For example, prestimulus alpha power during a challenging perceptual discrimination task was positively correlated with self-reports of unfocused attention (Macdonald, Mathan, \& Yeung, 2011). Likewise, in a repetitive driving simulation study carried out over a period of 5 days, episodes of mind-wandering (identified through responses to unpredictable experience-sampling probes) were characterized by increased alpha power (Baldwin et al., 2017). On the other hand, in a study using experience-sampling probes of mind-wandering during a reading comprehension task, prestimulus alpha was not associated with self-reports of mind-wandering (Broadway, Franklin, \& Schooler, 2015). Together, these mixed findings imply a need for additional study examining the relationship between alpha and mind-wandering across different task contexts.

The present study sought to test the association between alpha oscillations and mind-wandering during a commonly used task of attention and cognitive control - the Stroop task. Experience-sampling probes, querying whether the participant was mind-wandering just prior to the probe, were occasionally interspersed during a single session of the task involving hundreds of trials. Using a within-subjects design, we contrasted EEG activity in windows of time preceding the probes as a function of whether the participant indicated mind-wandering in response to the probe. This allowed us to test the hypothesis that alpha activity is higher during periods of mind-wandering compared with periods of on-task cognition.

\section{Method}

\section{Participants}

Participants were 62 undergraduates (33 female, 25 male, four other/unreported gender) prescreened to exclude those who were color-blind or nonnative English speakers. All participants gave written informed consent, and the procedures were approved by the Haverford College Institutional Review Board.

The data were collected as part of a more comprehensive study examining neural aspects of error-related cognitive control in the Stroop task. The primary results of the study pertaining to error-related EEG patterns, associated pupil diameter, and performance are reported elsewhere (Compton et al., 2019). That separate report does not address any questions about mind-wandering, which is the focus of this report.

\section{Task}

Participants completed a six-choice Stroop task, controlled by E-Prime software. The task was to identify the font color (red, orange, yellow, green, blue, purple) of each stimulus word while ignoring the word meaning. Responses were made via key press using the first three fingers of each hand in normal typing position. One-third of the trials were congruent (word and color match), one-third were incongruent (word and color mismatch), and one-third were neutral (e.g., word "mouse" in red font).

On each trial, the stimulus word was presented for $150 \mathrm{~ms}$, followed by a blank screen until the participant's key press, and then by a 1,280-ms blank-screen intertrial interval. The task included 864 Stroop trials, divided into 12 blocks of 72 randomly ordered trials each, with brief self-paced breaks between blocks. The 12 experimental blocks were preceded by 12 practice trials that gave trial-wise feedback to ensure that participants learned the color-response mapping. The entire task lasted approximately 30 minutes.

Experience-sampling probes were placed randomly within each half-block of the task, for a total of 24 probes (two per block). The probe screen asked the participant to indicate with a key press whether, just prior to the probe, the participant was on task, mind-wandering, or a mix of both. On-screen instructions prior to the experimental blocks defined on task as 
meaning that "you were focused on the task and not thinking about anything unrelated to the task." Mind-wandering was defined as meaning "you were thinking about something completely unrelated to the task," such as "what to eat for dinner" "plans with friends," or "thoughts about an upcoming test" (adapted from Seli, Cheyne, \& Smilek, 2013). Both was defined as "you were thinking about two things at once: BOTH the task and something else unrelated to the task. For example, perhaps you were devoting enough of your attention to the task to complete it, but you had 'spare resources' to also think about something else entirely at the same time. We will refer to these situations as BOTH to refer to a mix of being partly on task and partly mind-wandering." The probe response option of Both was included to allow for the possibility that participants judge themselves to be sufficiently attentive to the task and yet still mind-wandering, a state that can occur when a task becomes more automated. After the participant selected one of these options via a key press in response to the probe, a $1,280-\mathrm{ms}$ blank screen intervened before the next stimulus onset.

\section{EEG data acquisition}

EEG was recorded using a NuAmps amplifier controlled by Curry software. The following scalp sites, using a QuikCaps elastic cap and $\mathrm{Ag} / \mathrm{AgCl}$ electrodes, were sampled at $1000 \mathrm{~Hz}$ : $\mathrm{F} 3, \mathrm{Fz}, \mathrm{F} 4, \mathrm{FCz}, \mathrm{C} 3, \mathrm{Cz}, \mathrm{C} 4, \mathrm{P} 3, \mathrm{Pz}, \mathrm{P} 4, \mathrm{PO} 1, \mathrm{PO} 2$, in addition to eye leads (at the left and right temples and above and below the left eye) and left and right mastoids. The right mastoid was used as the reference at the time of recording. Off-line, EEG data were filtered with a $1-30 \mathrm{~Hz}$ bandpass filter and rereferenced to the average of the left and right mastoids.

Because experience-sampling probe onsets and probe responses were marked in the continuous EEG data file, we could identify windows of time preceding probes in which the response to the probe was either on task (OT), mindwandering (MW), or Both. For the primary analysis, the continuous file was segmented into 5 -sec epochs preceding each probe. Because mind-wandering is spontaneous in both its onset and offset, it has no fixed duration nor fixed temporal spacing in relation to an unexpected probe onset. Thus, 5 seconds was chosen arbitrarily as a preprobe time window that seemed likely to capture the momentary nature of mindwandering while still allowing sufficient EEG data for robust analysis. Subsequently, at the suggestion of peer reviewers, additional analyses examined preprobe epochs of 2 and 10 seconds, as well as a 5 -sec epoch spanning from 5 to 10 seconds before the probe, to further explore the limits of the association between preprobe alpha power and the participant's response to the probe.

EEG power spectra were calculated using the fast Fourier transform for each preprobe epoch, and power spectra were averaged separately for epochs corresponding to each probe- response category. Mean log alpha power (defined as 8-12 $\mathrm{Hz}$ ) was then extracted for each participant and proberesponse category.

\section{Self-report measures}

Immediately following the Stroop task, the participant completed a series of self-report questionnaires via an online survey. Participants used a sliding scale to estimate the percentage of the time during the task in which their mind was wandering. Next, the participant answered the five items of the Mind-Wandering Questionnaire (MWQ; Mrazek, Phillips, Franklin, Broadway, \& Schooler, 2013), which is intended to tap dispositional tendencies toward task-unrelated thought. As sample item is: "While reading, I find I haven't been thinking about the text and must therefore read it again." The MWQ uses a 6-point Likert-type scale, with response options ranging from almost never to almost always. Participants subsequently completed three additional questionnaires that are not further examined here (Positive and Negative Affect Schedule: Watson, Clark, \& Tellegen, 1988; Five Facet Mindfulness Questionnaire: Baer et al., 2008; Mood and Anxiety Symptom Questionnaire: Wardenaar et al., 2010).

\section{Results}

\section{Probe response data}

As described in the Method section, participants categorized their experience (OT, MW, or Both) in response to each of 24 experience-sampling probes. The mean number of responses (counts) in each of these categories was $M=10.8$ for OT, $M=$ 5.7 for $\mathrm{MW}$, and $M=7.5$ for Both. Individual participants varied widely in their counts in each category (range across participants, 0-23 for OT responses, 0-24 for MW responses, and $0-16$ for Both responses). As a result, it was not possible to compare EEG data preceding each of the three probe response categories for all participants, because some participants had no instances of a particular category. To maximize the number of participants who could be included in EEG analyses while still maintaining meaningful categories, we summed probe counts across the MW and Both categories to create a combined category of responses (hereafter referred to as MWB), in which any degree of mind-wandering was reported. We then included for subsequent EEG analysis only those participants who had at least five instances of OT responses and five instances of MWB responses. This selection procedure resulted in 50 participants in whom the OT-MWB contrast could be carried out within subjects. For this subsample, the mean number of probe responses was $M=11.5$ for OT responses and $M=12.5$ for MWB responses. (An alternative participant-selection procedure that required at least seven 
trials of each type yielded nearly identical statistical outcomes in the analysis of EEG data from the 39 participants who would be included under those stricter criteria.)

\section{Performance data}

Mean accuracy on the Stroop task was $91.7 \%$ correct. Typical Stroop interference effects were evident in accuracy, $F(2,122)$ $=12.02, p<.001$ (congruent $M=93.0 \%, S E M=0.7 \%$, incongruent $M=88.6 \%, S E M=1.6 \%$, neutral $M=93.5 \%, S E M$ $=0.7 \%)$ and in reaction time on correct trials, $F(2,122)=$ 143.79, $p<.001$ (congruent $M=615 \mathrm{~ms}, S E M=18 \mathrm{~ms}$, incongruent $M=698 \mathrm{~ms}, S E M=19 \mathrm{~ms}$, neutral $M=597$ $\mathrm{ms}, S E M=17 \mathrm{~ms})$.

Stroop-task performance in the two trials preceding probes was examined as a function of probe response category to examine whether episodes of mind-wandering were associated with decrements in performance. A two-trial preprobe window was selected because it approximately matches the 5second preprobe window of EEG data. This analysis was conducted on the 50 participants meeting criteria of at least five OT and five MWB responses. Accuracy was slightly but nonsignificantly lower in the two Stroop trials preceding MWB probe responses $(M=88.0 \%$, SEM $=1.9 \%)$ compared with OT probe responses $(M=90.4 \%, S E M=1.4 \%), t(49)=1.46$, $p=.152, d=.206$. Likewise, responses were nonsignificantly slower on the two Stroop trials preceding MWB probe responses $(M=656 \mathrm{~ms}, S E M=24 \mathrm{~ms})$ compared with OT probe responses $(M=617 \mathrm{~ms}, S E M=22 \mathrm{~ms}), t(49)=-1.90, p=$ $.064, d=.268$. Comparisons of preprobe behavior yielded similar conclusions when only one trial prior to the probe was considered (accuracy: pre-MWB $M=87.4 \%$, pre-OT $M$ $=89.7 \%$ ), $t(49)=1.06, p>.29$ (reaction time: pre-MWB $M=$ $663 \mathrm{~ms}$, pre-OT $M=613 \mathrm{~ms}), t(49)=-1.77, p<.09$. Restricting analyses to behavior preceding an OT or MW response, rather than comparing OT with combined MWB responses, did not yield significant preprobe performance differences ( $p s>.40$; restricted to $n=24$ participants with at least five OT and at least five MW probe responses).

\section{Retrospective self-report data}

We examined whether retrospective self-report measures of mind-wandering were correlated with experience-sampling probe responses. For two participants, retrospective data were unavailable due to experimenter error. For the remaining 60 participants, the retrospective estimate of time spent mindwandering during the Stroop task $(M=45.9 \%, S D=19.9 \%)$ was negatively correlated with the OT probe response count $(r$ $=-0.70, p<.001$ ), positively correlated with the MW probe response count $(r=.66, p<.001)$, and marginally correlated with the Both probe response count $(r=.23, p<.09)$. Thus, participants' retrospective estimate immediately following the task tended to match their responses to the experience sampling probes during the task. In contrast, scores on the 5-item MWQ, which is intended to tap trait-like aspects of mindwandering, were not significantly correlated with either the probe response counts nor the retrospective estimate of mind-wandering during the task ( $p s>.15$ ).

\section{EEG data}

The next step in the analyses addressed the main hypothesis by comparing EEG alpha power values for the 5-second interval just prior to probes as a function of the participant's response to the probe. Log alpha power values were submitted to a $2 \times 12$ repeated-measures ANOVA, with probe response type (OT, MWB) and electrode site as factors. The main effect of probe response type, $F(1,49)=20.98, p<.001, \eta_{\mathrm{p}}{ }^{2}=0.30$, was due to higher alpha power preceding probes that elicited MWB responses $\left(M=0.97 \mu \mathrm{V}^{2}, S E M=0.05\right)$ compared with probes that elicited OT responses $\left(M=0.91 \mu \mathrm{V}^{2}, S E M=\right.$ $0.05)$. The main effect of site, $F(11,539)=14.02, p<.001$, $\eta_{\mathrm{p}}{ }^{2}=0.22$, and the Probe Response Type $\times$ Site interaction, $F(11,539)=5.51, p<.005, \eta_{\mathrm{p}}^{2}=.10$, generally reflected higher alpha power toward posterior sections of the scalp, and a greater OT-MWB probe response differentiation toward posterior sections of the scalp. Fig. 1 shows means for the interaction.

While the primary analysis focused on alpha power in a 5second preprobe epoch, additional analyses were conducted on 2 -second and 10-second preprobe epochs on an exploratory

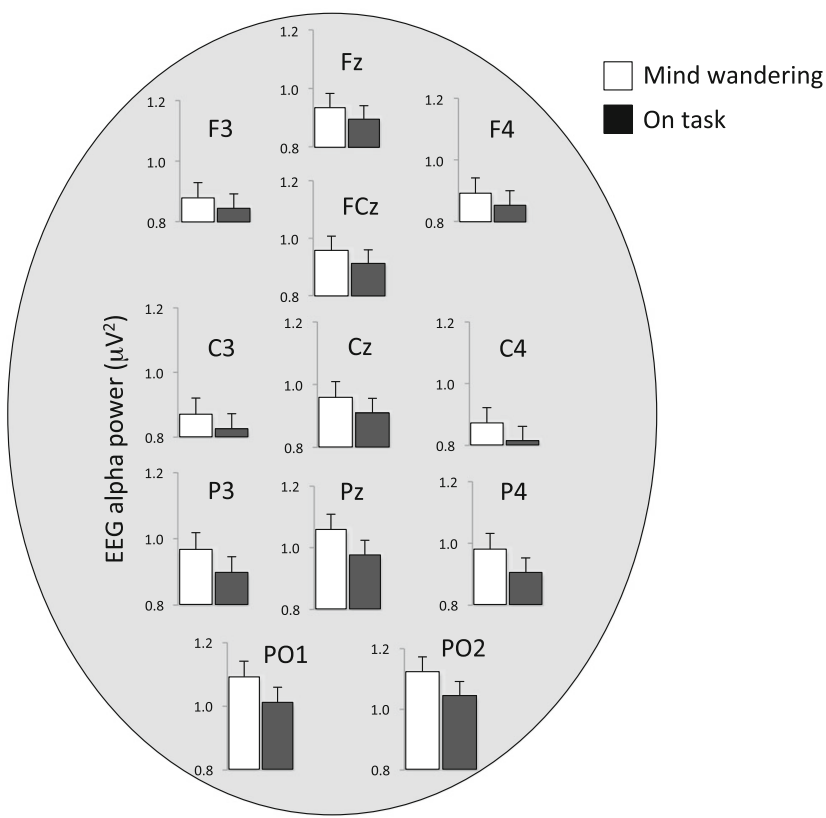

Fig. 1 EEG alpha power during the 5-second window preceding experience-sampling probes, separately for probes to which participants indicated mind-wandering versus on-task cognition. Results are displayed separately for 12 electrode sites represented schematically on the scalp 
basis. For each analysis, a $2 \times 12$ (Probe Response Type $\times$ Electrode Site) repeated-measures ANOVA was conducted on alpha power. The main effect of probe response type was significant when the preprobe epoch was 2 seconds, $F(1,49)=$ $11.68, p<.001, \eta_{\mathrm{p}}{ }^{2}=0.19$ (pre-MWB $M=2.46 \mu \mathrm{V}^{2}$, pre-OT $M=2.30 \mu \mathrm{V}^{2}$ ) and when the preprobe epoch was 10 seconds, $F(1,49)=40.56, p<.001, \eta_{\mathrm{p}}{ }^{2}=0.45$ (pre-MWB $M=0.66$ $\mu \mathrm{V}^{2}$, pre-OT $M=0.61 \mu \mathrm{V}^{2}$ ). Thus, across each of the three epochs lengths (2, 5, and 10 seconds before the probe), preprobe alpha power was significantly higher preceding a MWB compared with an OT response to the probe. Furthermore, analysis on data extracted from the epoch from -5 to -10 seconds in relation to the probe found again a main effect of probe response type, $F(1,49)=33.44, p<.001, \eta_{\mathrm{p}}{ }^{2}=$ 0.41 , again with higher alpha preceding a MWB response $(M=$ $\left.0.97 \mu \mathrm{V}^{2}\right)$ compared with an OT response $\left(M=0.90 \mu \mathrm{V}^{2}\right)$. Direct comparison of data from the 0 -s to -5 -s preprobe epoch versus the -5 -s to -10 -s preprobe epoch, in an ANOVA with epoch, probe response type, and site found again a main effect of probe response type, $F(1,49)=39.17, p<.001, \eta_{\mathrm{p}}{ }^{2}=0.44$, that did not further interact with epoch $(F<1)$.

Although statistical analyses focused on the alpha frequency band, for which prior literature supports a rationale for hypothesizing effects related to mind-wandering, Fig. 2 presents the power spectrum for all measured EEG frequencies at a representative site (Pz). As evident in the Fig. 2, the 8-12 Hz alpha frequency band distinguishes between on-task and mind-wandering episodes while other frequencies generally do not.

\section{Exploratory correlational analyses}

Exploratory analyses examined correlations among variables measured in the preprobe window, to address whether participants with greater alpha differentiation between MWB and OT responses would also show greater performance differentiation prior to the probes. First, for alpha power data, we subtracted alpha power in the 5 -s window preceding OT probe responses from alpha power preceding MWB probe responses. This subtraction creates a probe-response difference score in which a higher score means relatively more alpha preceding MWB probe responses compared with OT probe responses. This alpha probe-response difference score was correlated with performance-based differences scores. We computed two behavioral indices: Accuracy 2 trials previous to an OT probe response minus Accuracy 2 trials previous to a MWB probe responses (accuracy probe-response difference score), and Reaction Time 2 trials previous to MWB probe responses minus Reaction Time 2 trials previous to an OT probe response (reaction-time probe-response difference score).

The alpha-based probe-response difference score was not significantly related to the reaction-time probe-response

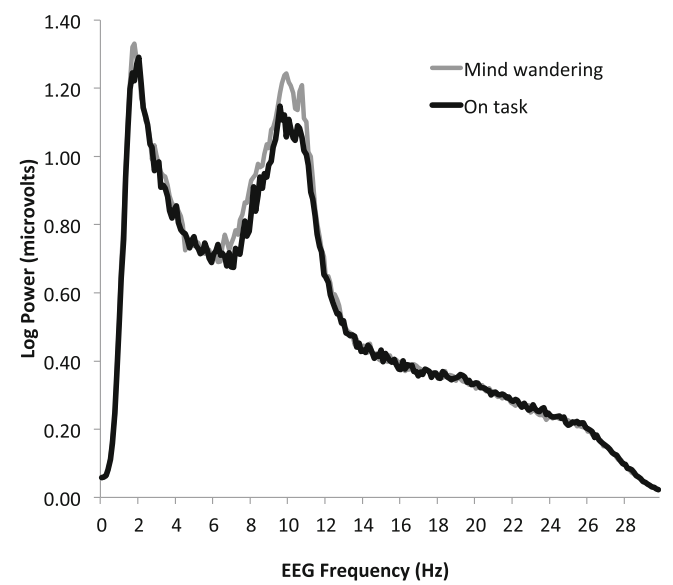

Fig. 2 EEG power (in $\mu V^{2}$ ) across all measured frequencies in the 5second window prior to experience-sampling probes. Data are represented separately for probes to which participants indicated some degree of mind-wandering versus being fully on task. Data are taken from the $\mathrm{Pz}$ electrode site. Mind-wandering versus on-task episodes are best distinguished by frequencies in the alpha range $(8-12 \mathrm{~Hz})$

difference score, but unexpectedly it was inversely related to the accuracy probe-response difference score $(r=-0.327, p<$ $.05)$. This inverse relationship remained significant even when mean accuracy and mean alpha power were statistically controlled in a partial correlation (partial $r=-.341, p<.02, d f=$ 46). In sum, somewhat surprisingly, the participants whose alpha power in the preprobe epoch best differentiated between MWB versus OT probe responses were those whose accuracy was least detrimentally affected by MWB versus OT probe responses.

\section{Discussion}

This study combined experience-sampling probes and EEG recording to identify neural signatures associated with mindwandering. During a repetitive and lengthy Stroop task, participants indicated some degree of mind-wandering in response to about half of the randomly placed experience sampling probes, and they retrospectively estimated that they spent an average of $46 \%$ of their task time mind-wandering. The key finding of this study was that in a 5-s window of time preceding an experience sampling probe, EEG alpha power was higher if participants indicated some degree of mindwandering instead of being fully on task prior to the probe. The same pattern of results was obtained when the preprobe epoch was shortened to 2 seconds or increased to 10 seconds. Furthermore, EEG data within an epoch 5 to 10 seconds before the probe was equally predictive of a mind-wandering response as data within an epoch 0 to 5 seconds before the probe. Although task parameters precluded analyzing even longer preprobe epochs, future studies could further explore 
how long before a self-report of mind-wandering the increased alpha patterns are evident.

The results provide strong evidence of an association between mind-wandering and EEG alpha activity, consistent with prior theory and research associating alpha oscillations with internally directed cognition (e.g., Adrian \& Mathews, 1934; Thut et al., 2006). As such, the results complement other findings of reduced neural indices of attention toward incoming stimuli during episodes of mind-wandering (Barron, Riby, Greer, \& Smallwood, 2011; Handy \& Kam, 2015; Smallwood, Beach, Schooler, \& Handy, 2008). That is, the present data imply that mind-wandering is associated with increases in neural measures assumed to reflect inward-directed cognition, while prior studies imply that it is also associated with decreases in neural measures assumed to reflect externally driven cognition. Recent work found that a machine learning algorithm that combines oscillatory EEG measures such as alpha power with ERP indices of externally driven attention was able to predict the presence of a mind-wandering state at above-chance levels, with alpha power serving as the most reliable individual predictor (Jin, Borst, \& van Vugt, 2019).

These results are consistent with prior findings associating higher alpha power with mind-wandering or attentional lapses (Baldwin et al., 2017; Macdonald et al., 2011), while standing in contrast to studies that found the opposite pattern (Braboszcz \& Delorme, 2011) or no difference in alpha power depending on self-reported mind-wandering (Broadway et al., 2015). Given differences in methodology among these studies, the critical variables contributing to different outcomes have yet to be fully identified. However, because the only study to directly contradict the current findings is one that used a primary task of internally directed attention (breathcounting; Braboszcz \& Delorme, 2011), it seems likely that alpha power provides a better index of off-task thought when the primary task involves responding to external stimuli, as in the present study and others that produced similar results (Baldwin et al., 2017; McDonald et al., 2011).

Although Stroop performance was also slightly worse preceding mind-wandering versus on-task probe responses, performance measures did not distinguish between mindwandering versus on-task experience as robustly as EEG alpha power did. One possibility is that this EEG-versusperformance difference is due to measurement issues: EEG data are more fine-grained, with 5,000 data points per electrode during a 5-second interval, as opposed to two button presses within that same approximate time interval. Thus, perhaps performance measures are subject to more random noise and therefore less able to demonstrate effects of the mental state of mind-wandering.

A second, and possibly more interesting, possibility is that EEG measures provide a more direct window onto mindwandering than do performance measures, reflecting the process of mind-wandering itself rather than the effects of that process on other aspects of cognition. As a task becomes more practiced and automated, its demand on cognitive resources decreases, and participants may be able to sustain sufficient levels of performance while still allowing their minds to wander (Thomson, Besner, \& Smilek, 2013). Indeed, in the present study, participants maintained a high accuracy rate $(92 \%$ correct) across more than 800 trials of a challenging six-choice Stroop task even while self-reporting that their minds wandered almost half the time. Particularly for overpracticed tasks or for participants with high cognitive capacity, EEG measures may index a mind-wandering attentional state that is not as easily discernible with overt performance measures alone. This interpretation is supported by the intriguing yet unexpected finding that participants whose alpha power best distinguished self-reported mind-wandering from on-task attentional states were those whose performance accuracy was least disrupted by mind-wandering.

While the robust results imply a close connection between alpha oscillations and mind-wandering experiences, they leave the causal nature of the relationship open to future research. Additional studies could systematically vary task conditions known to elicit mind-wandering (such as motivation or cognitive load) and examine how alpha power changes as a result. Conversely, researchers have used $10-\mathrm{Hz}$ rhythmic transcranial magnetic stimulation of the brain to cause perceptual changes previously correlated with alpha rhythms (Romei, Gross, \& Thut, 2010; Thut, Schyns, \& Gross, 2011). Using a similar method, future studies could test whether stimulation in the alpha frequency band can elicit episodes of mind-wandering (cf. Clayton, Yeung, \& Cohen Kadosh, 2019). In addition, while the present study focused primarily on alpha rhythms, because of the robust prior literature motivating hypotheses about this measure, other studies may choose to expand beyond alpha to examine other oscillatory EEG phenomena (e.g., van Son et al., 2019).

Future studies may also examine the relationships among pupil dilation, EEG alpha power, and mind-wandering. Prior research has found that pupil diameter covaries with mindwandering states (e.g., Unsworth \& Robison, 2016, 2017; see also Mittner et al., 2014), possibly reflecting a role of the norepinephrinergic system in attentional fluctuations (Mittner, Hawkins, Boekel, \& Forstmann, 2016). Although the present study collected pupillary measurements, those measurements were only obtained for the Stroop trials in the present study, not the probe response trials, because the aim was to examine pupillary reactions following Stroop performance errors (Compton et al., 2019). Thus, associations between pupil dilation and mind-wandering could not be directly examined within the present data set. However, other findings indicate that EEG alpha power and pupil dilation are inversely related (Compton et al., 2019), possibly reflecting a common arousal system and opening possibilities for further investigation of joint pupillary-EEG predictions of mind-wandering states. 
While the main results of the study are statistically robust and support the hypothesis that internally directed thought is characterized by enhanced alpha rhythms, one limitation of the study involves the nature of the mind-wandering probes. It is challenging to define and measure a mental state that is, by definition, spontaneous and internally experienced (Smallwood \& Schooler, 2015). While randomly placed experience-sampling probes have advantages as a method of evaluating the presence of mind-wandering, their usefulness is limited by the response options available as well as by participants' ability to introspect about the states being probed. In the present study, participants were given three response choices (on task, mind-wandering, or both), which may not sufficiently differentiate among related, but distinct mental states. For example, some prior findings have distinguished between intentional and unintentional mind-wandering (Seli, Risko, \& Smilek, 2016; Seli, Risko, Smilek, \& Schacter, 2016), and others have differentiated among states of task-related interference, mind-wandering, and external distraction (e.g., Stawarczyk, Majerus, Maquet, \& D'Argembeau, 2011). In the present study, the response option of "both" was intended to capture states in which participants divided attention between the primary task and internally generated thoughts; however, because of the uneven distribution of responses across the three probe response options, it was necessary to merge the "both" responses with the mind-wandering responses to achieve sufficient trials for analysis. Future research would benefit from considering additional ways to parse the concept of mindwandering and related experiences, which could in turn lead to more specific interpretations of the role of alpha rhythms in supporting experiences of internally directed thought.

Acknowledgements Paige Carson, Elizabeth Heaton, Stephanie Histon, Taylor Levine, and Danielle Rette assisted with data collection. This study was supported by NSF RUI Grant No. 1632584.

Open practices statement The data from this study are available from the first author upon request. The study was not preregistered.

\section{References}

Adrian, E. D., \& Matthews, B. H. (1934). The Berger rhythm: Potential changes from the occipital lobes in man. Brain, 57(4), 355-385.

Andrews-Hanna, J. R., Irving, Z. C., Fox, K. C., Spreng, R. N., \& Christoff, K. (2018). The neuroscience of spontaneous thought: An evolving interdisciplinary field. In K.C. Fox \& K. Christoff (Eds.), The Oxford handbook of spontaneous thought: Mind-wandering, creativity, and dreaming. Oxford, UK: Oxford University Press. https://doi.org/10.1093/oxfordhb/9780190464745.013.33

Baer, R. A., Smith, G. T., Lykins, E., Button, D., Krietemeyer, J., Sauer, S., . . Williams, J. M. (2008). Construct validity of the Five Facet Mindfulness Questionnaire in meditating and nonmeditating samples. Assessment, 15(3), 329-342.

Baldwin, C. L., Roberts, D. M., Barragan, D., Lee, J. D., Lerner, N., \& Higgins, J. S. (2017). Detecting and quantifying mind wandering during simulated driving. Frontiers in Human Neuroscience, 11, 406. https://doi.org/10.3389/fnhum.2017.00406

Barron, E., Riby, L. M., Greer, J., \& Smallwood, J. (2011). Absorbed in thought: The effect of mind wandering on the processing of relevant and irrelevant events. Psychological Science, 22(5), 596-601.

Braboszcz, C., \& Delorme, A. (2011). Lost in thoughts: Neural markers of low alertness during mind wandering. NeuroImage, 54(4), 3040 3047.

Broadway, J. M., Franklin, M. S., \& Schooler, J. W. (2015). Early eventrelated brain potentials and hemispheric asymmetries reveal mindwandering while reading and predict comprehension. Biological Psychology, 107, 31-43.

Carp, J., \& Compton, R. J. (2009). Alpha power is influenced by performance errors. Psychophysiology, 46(2), 336-343.

Clayton, M. S., Yeung, N., \& Cohen Kadosh, R. (2019). Electrical stimulation of alpha oscillations stabilizes performance on visual attention tasks. Journal of Experimental Psychology: General, 148(2), 203-220.

Compton, R. J., Arnstein, D., Freedman, G., Dainer-Best, J., \& Liss, A. (2011). Cognitive control in the inter-trial interval: Evidence from EEG alpha power. Psychophysiology, 48, 583-590.

Compton, R. J., Rette, D., Gearinger, D., Wild, H., Histon, S., Heaton, E., \& Thiel, P. (2019). Post-error arousal: Simultaneous EEG and pupillary responses to performance errors. Manuscript in preparation.

Cooper, N. R., Croft, R. J., Dominey, S. J., Burgess, A. P., \& Gruzelier, J. H. (2003). Paradox lost? Exploring the role of alpha oscillations during externally vs. internally directed attention and the implications for idling and inhibition hypotheses. International Journal of Psychophysiology, 47(1), 65-74.

Gruberger, M., Ben-Simon, E., Levkovitz, Y., Zangen, A., \& Hendler, T. (2011). Towards a neuroscience of mind-wandering. Frontiers in Human Neuroscience, 5, 56. https://doi.org/10.3389/fnhum.2011. 00056

Handy, T. C., \& Kam, J. W. Y. (2015). Mind wandering and selective attention to the external world. Canadian Journal of Experimental Psychology/Revue canadienne de psychologie expérimentale, 69(2), 183-189.

Jin, C. Y., Borst, J. P., \& van Vugt, M. K. (2019). Predicting task-general mind-wandering with EEG. Cognitive, Affective, \& Behavioral Neuroscience, 1-15. Advance online publication. https://doi.org/ 10.3758/s13415-019-00707-1

Knyazev, G. G., Slobodskoj-Plusnin, J. Y., Bocharov, A. V., \& Pylkova, L. V. (2011). The default mode network and EEG alpha oscillations: An independent component analysis. Brain Research, 1402, 67-79.

Macdonald, J. S. P., Mathan, S., \& Yeung, N. (2011). Trial-by-trial variations in subjective attentional state are reflected in ongoing prestimulus EEG alpha oscillations. Frontiers in Psychology, 2, 82. https://doi.org/10.3389/fpsyg.2011.00082

Mittner, M., Boekel, W., Tucker, A. M., Turner, B. M., Heathcote, A., \& Forstmann, B. U. (2014). When the brain takes a break: A modelbased analysis of mind wandering. Journal of Neuroscience, 34(49), 16286-16295.

Mittner, M., Hawkins, G. E., Boekel, W., \& Forstmann, B. U. (2016). A neural model of mind wandering. Trends in Cognitive Sciences, 20(8), 570-578.

Mo, J., Liu, Y., Huang, H., \& Ding, M. (2013). Coupling between visual alpha oscillations and default mode activity. NeuroImage, 68,112 118.

Mrazek, M. D., Phillips, D. T., Franklin, M. S., Broadway, J. M., \& Schooler, J. W. (2013). Young and restless: Validation of the Mind-Wandering Questionnaire (MWQ) reveals disruptive impact of mind-wandering for youth. Frontiers in Psychology, 4, 560. https://doi.org/10.3389/fpsyg.2013.00560

Romei, V., Gross, J., \& Thut, G. (2010). On the role of prestimulus alpha rhythms over occipito-parietal areas in visual input regulation: 
Correlation or causation? Journal of Neuroscience, 30(25), 86928697.

Seli, P., Cheyne, J. A., \& Smilek, D. (2013). Wandering minds and wavering rhythms: Linking mind wandering and behavioral variability. Journal of Experimental Psychology: Human Perception and Performance, 39(1), 1-5. Advance online publication. https://doi. org/10.1037/a0030954

Seli, P., Risko, E. F., \& Smilek, D. (2016). On the necessity of distinguishing between unintentional and intentional mind wandering. Psychological Science, 27(5), 685-691.

Seli, P., Risko, E. F., Smilek, D., \& Schacter, D. L. (2016). Mindwandering with and without intention. Trends in Cognitive Sciences, 20(8), 605-617.

Smallwood, J., Beach, E., Schooler, J. W., \& Handy, T. C. (2008). Going AWOL in the brain: Mind wandering reduces cortical analysis of external events. Journal of Cognitive Neuroscience, 20(3), 458-469.

Smallwood, J., \& Schooler, J. W. (2015). The science of mind wandering: Empirically navigating the stream of consciousness. Annual Review of Psychology, 66, 487-518.

Stawarczyk, D., Majerus, S., Maquet, P., \& D’Argembeau, A. (2011). Neural correlates of ongoing conscious experience: Both taskunrelatedness and stimulus-independence are related to default network activity. PLOS ONE, 6(2), e16997.doi:https://doi.org/10.1371/ journal.pone.0016997

Thomson, D. R., Besner, D., \& Smilek, D. (2013). In pursuit of off-task thought: Mind wandering-performance trade-offs while reading aloud and color naming. Frontiers in Psychology, 4, 360. https:// doi.org/10.3389/fpsyg.2013.00360

Thut, G., Nietzel, A., Brandt, S. A., \& Pascual-Leone, A. (2006). $\alpha$-Band electroencephalographic activity over occipital cortex indexes visuospatial attention bias and predicts visual target detection. Journal of Neuroscience, 26(37), 9494-9502.

Thut, G., Schyns, P., \& Gross, J. (2011). Entrainment of perceptually relevant brain oscillations by non-invasive rhythmic stimulation of the human brain. Frontiers in Psychology, 2, 170. https://doi.org/10. 3389/fpsyg.2011.00170

Unsworth, N., \& Robison, M. K. (2016). Pupillary correlates of lapses of sustained attention. Cognitive, Affective, \& Behavioral Neuroscience, 16(4), 601-615.

Unsworth, N., \& Robison, M. K. (2017). The importance of arousal for variation in working memory capacity and attention control: A latent variable pupillometry study. Journal of Experimental Psychology: Learning, Memory, and Cognition, 43(12), 1962-1987.

van Dijk, H., Schoffelen, J. M., Oostenveld, R., \& Jensen, O. (2008). Prestimulus oscillatory activity in the alpha band predicts visual discrimination ability. Journal of Neuroscience, 28(8), 1816-1823.

van Son, D., De Blasio, F. M., Fogarty, J. S., Angelidis, A., Barry, R. J., \& Putman, P. (2019). Frontal EEG theta/beta ratio during mind wandering episodes. Biological Psychology, 140, 19-27.

Wardenaar, K. J., van Veen, T., Giltay, E. J., de Beurs, E., Penninx, B. W., \& Zitman, F. G. (2010). Development and validation of a 30 -item short adaptation of the Mood and Anxiety Symptoms Questionnaire (MASQ). Psychiatry Research, 179(1), 101-106.

Watson, D., Clark, L. A., \& Tellegen, A. (1988). Development and validation of brief measures of positive and negative affect: The PANAS scales. Journal of Personality and Social Psychology, 54(6), 1063-1070.

Publisher's note Springer Nature remains neutral with regard to jurisdictional claims in published maps and institutional affiliations. 